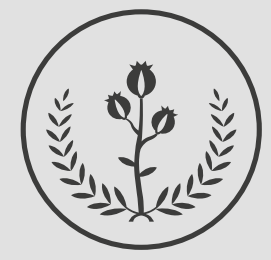

FUCS

\title{
Proyección del tiempo para alcanzar la inmunidad de rebaño para COVID-19 en Bogotá
}

\section{Tiime projection to reach herd immunity for COVID-19 in Bogotá}

a Ingeniero. Magister en Gestión de la Tecnología Educativa, Especialista en Administración de la Informática Educativa.

Docente de matemáticas e Investigador, Secretaría de Educación de Soacha, Cundinamarca.

\section{R E S U M E N}

Introducción: el 17 de febrero de 2021 comenzó el proceso de inmunización en el país basados en el Plan Nacional de Vacunación estructurado por el gobierno nacional, el cual establece dos fases y cinco etapas. Las vacunas que se aplicarán en Colombia son: Pfizer, AstraZeneca, Janssen, Moderna y Sinovac. Objetivo: calcular el tiempo para alcanzar la inmunidad de rebaño para COVID-19 en Bogotá. Metodología: la información de vacunación diaria se obtuvo de la Secretaría Distrital de Salud. Se tomó el período completo (21 de febrero 2021 al 11 de julio 2021) correspondiente a 140 días del inicio de la vacunación en Bogotá. Resultados: se determinó que, para el 6 de diciembre 2021, se logrará obtener la inmunidad de rebaño, para esta fecha se habrán aplicado 11'041.306 dosis para inmunizar a la población en Bogotá. Conclusión: de esta investigación se infiere el significativo papel que adquieren los modelos matemáticos a la hora de simular los procesos de vacunación y de esta manera, se esbozan a futuro vías de investigación en la modelización matemática para lograr la inmunidad de rebaño en cualquier proceso infeccioso.

Palabras clave: SARS-CoV-2, vacunas, inmunidad, modelos matemáticos.

(C) 2021 Fundación Universitaria de Ciencias de la Salud - FUCS. Este es un artículo Open Access bajo la licencia CC BY-NC-ND (http://creativecommons.org/licenses/by-nc-nd/4.0/).

\section{INFORMACIÓN DEL ARTÍCULO}

Historia del artículo:

Fecha recibido: julio 13 de 2021 Fecha aceptado: julio 28 de 2021

\footnotetext{
Autor para correspondencia.

Jorge Enrique Díaz Pinzón jediazp@unal.edu.co
}

DOI

10.31260/RepertMedCir.01217372.1252 


\section{A B S T R ACT}

Introduction: : the immunization process in the country started on February 17 2021, based on the National Vaccination Plan structured by the National Government, which establishes two phases and five stages. The vaccines to be applied in Colombia are: Pfizer, AstraZeneca, Janssen, Moderna and Sinovac. Objective: to estimate the time to achieve herd immunity for COVID-19 in Bogota. Methodology: daily vaccination information was obtained from the Bogota District Health Authority. The full 140-day period from February 212021 to July 112021 corresponding to the vaccination rollout in Bogota, was reviewed. Results: it was determined that herd immunity will be reached by December 6 2021. As of this date 11'041.306 doses will have been administered to immunize the population in Bogota. Conclusion: from this research we infer the significant role that mathematical models play when simulating vaccination processes, thus, future research avenues based on mathematical modeling to achieve herd immunity for any infectious process, will be outlined.

Key words: SARS-CoV-2, vaccines, immunity, mathematical models.

(C) 2021 Fundación Universitaria de Ciencias de la Salud - FUCS. This is an open access article under the CC BY-NC-ND license (http://creativecommons.org/licenses/by-nc-nd/4.0/)

INTRODUCCIÓN

A finales de diciembre 2019, en Wuhan (provincia de Hubei, China), se anuncia un conglomerado local de casos de neumonía. Después se establece que son causados por un nuevo coronavirus. ${ }^{1}$ La Organización Mundial de la Salud (OMS) declaró el 11 de marzo 2020 el estado de pandemia debido a la infección por SARS-CoV-2. El COVID-19 se propagó ampliamente tras la rápida diseminación del virus en China y comenzó a introducirse en otros países, reportándose, hasta el 10 de abril 2021, 134'719.328 casos y 2'915.972 muertes relacionadas de manera directa con el virus en el mundo. ${ }^{2}$ Las primeras medidas para prevenir la transmisión del virus se instituyeron en la separación y la protección mediante barreras físicas para evitar la dispersión de aerosoles. En el caso de personas con mayor exposición de riesgo, como los trabajadores de la salud, el abastecimiento y el uso adecuado de equipos de protección personal, fue el principal instrumento preventivo, junto al distanciamiento, lavado de manos y uso de alcohol en gel. ${ }^{3}$

En este momento se encuentran acreditadas para uso de emergencia las siguientes vacunas: CoviShield (AstraZeneca/ Oxford) de la firma AstraZeneca SA basada en un vector viral no replicativo (adenovirus de chimpancé); BNT162b2 de la firma Pfizer, se fundamenta en una plataforma de ácidos nucleicos (ARN mensajero); Sputnik V del Instituto Gamaleya basada en una plataforma de vector viral no replicativo (adenovirus humanos: 26 y 5) y por último; BBIBP-CorV de la firma Sinopharm que se fundamente en una plataforma de virus inactivado. ${ }^{4}$

La «inmunidad de rebaño" o «inmunidad colectiva», también conocida como «inmunidad de la población», es un concepto manejado para la vacunación, en el que una población puede preservarse de un determinado virus si se alcanza un umbral de vacunación. Todavía estamos aprendiendo sobre la inmunidad al COVID-19. La mayoría de las personas que están contagiadas acrecientan una respuesta inmune dentro de las primeras semanas, pero no sabemos qué tan fuerte o perdurable es esa respuesta ni en qué se diferencia para diferentes personas. También hay informes de personas infectadas con COVID-19 por segunda vez. "El umbral de inmunidad de rebaño depende del número de reproducción básica (R0) y se define como $1-1 /$ R0. Mientras más contagioso sea un patógeno mayor será su R0 y mayor la proporción de la población que deberá ser inmune para poder bloquear la transmisión en forma sostenida. El umbral de inmunidad de rebaño puede variar entre diferentes poblaciones ya que el R0 dependerá de diversos factores, como la densidad y la estructura de una población". ${ }^{6}$ En el caso de SARS-CoV-2, el R0 se ha estimado en 3,28, aunque puede variar dependiendo de múltiples factores. En el caso de SARS-CoV-2 se ha considerado que esta inmunidad de rebaño surge cuando más de $70 \%$ de las personas están protegidas. ${ }^{7}$ El 17 de febrero 2021 comenzó el proceso de inmunización en el país basados en el plan nacional de vacunación estructurado por el gobierno nacional, el cual establece dos fases $y$ cinco etapas. En la primera etapa se contempla la primera línea del talento humano en salud y adultos mayores de 80 años. Las vacunas que se aplican en Colombia son: Pfizer, AstraZeneca, Janssen, Moderna y Sinovac. ${ }^{8}$ De acuerdo con la información anterior el presente trabajo tiene por objetivo calcular el tiempo para alcanzar la inmunidad de rebaño para COVID-19 en Bogotá, a partir de la fase 1 y las etapas 1 y 2 de vacunación, teniendo como priorización los grupos en riesgo. La población proyectada para Bogotá en 2021, según el DANE es de 7'834.167 personas, y así para alcanzar la inmunidad de rebaño se necesita que 5'483.917 millones de bogotanos estén vacunados, según la información de la Secretaría Distrital de Salud de Bogotá ${ }^{9}$ (figura 1). 


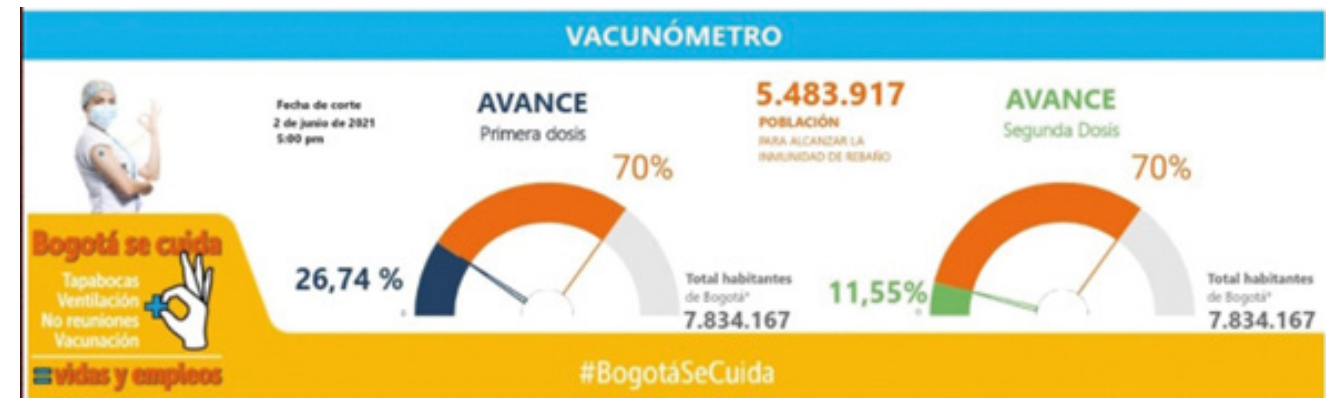

Figura 1. Alcance de la vacunación contra el COVID-19 en Bogotá. Fuente: Secretaría Distrital de Salud. ${ }^{9}$

\section{Modelo ARIMA (p,d,q)}

El modelo ARIMA (Autoregresive Integrated Moving Average) admite referir un valor como una función lineal de datos anteriores y errores convenidos al azar y puede contener un componente cíclico o estacional. ${ }^{10,11}$ Los modelos ARIMA se erigen a partir de los modelos ARMA, pero estimando que la serie en estudio para que sea estacionaria en media, tendrá que diferenciarse una serie de veces. Un modelo ARIMA (p, d, q) es un modelo $\operatorname{ARMA~(p,~q)~sobre~la~}$ serie diferenciada d veces. Es decir, su expresión algebraica, será:

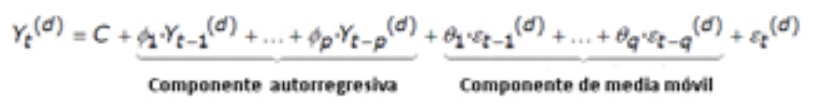

donde $\mathrm{Yt}^{(\mathrm{d})}$ es la serie de las diferencias de orden $\mathrm{d}$ y $\varepsilon \mathrm{t}^{(\mathrm{d})}$ es la serie de los errores que se cometen en la serie anterior. ${ }^{12}$ Regularmente el orden de diferenciación d, entero, oscila entre 0 y 2. Una vez hemos visto el procedimiento de un modelo ARIMA, podemos afirmar que este se puede precisar como un modelo de regresión lineal múltiple, donde la variable dependiente es la propia serie (diferenciada o no) y las variables independientes son valores de la serie y valores de los errores de ajuste pasados hasta unos órdenes $\mathrm{p} \mathrm{y} \mathrm{q,}$ comparativamente. ${ }^{12}$ El objetivo de los métodos de serie de tiempo es revelar un patrón en los datos históricos y luego extrapolarlo hacia el futuro; el pronóstico se cimenta solo en valores pasados de la variable que tratamos de predecir. ${ }^{13}$

\section{METODOLOGÍA}

El trabajo de investigación se realizó mediante un tipo experimental que es el siguiente: “aquella que permite mayor seguridad al establecer relaciones de causa a efecto pues presenta una visión general y aproximada del objeto de estudio, además de contar con una investigación cuyo diseño establece un método experimental habitual del conjunto de las normas científicas", Monje (2011) citado por Díaz. ${ }^{14}$ Según Shuttleworth citado por Díaz, ${ }^{15,16}$, menciona que "regularmente a estos experimentos se les nombra ciencia verdadera y manejan medios matemáticos y estadísticos cotidianos para evaluar los resultados de modo concluyente".

\section{DATOS}

La información de vacunación diario se obtuvo de la Secretaría Distrital de Salud. Se tomó el período completo (21 de febrero 2021 a 11 de julio 2021) correspondiente a 140 días del inicio de la vacunación en Bogotá, para analizar la evolución de casos diarios de vacunación para primera dosis.

\section{ANÁLISIS ESTADÍSTICO}

Se llevó a cabo con el software de análisis estadístico SPSS versión 25. Utilizando el modelo lineal ARIMA se realiza la proyección de los resultados del número de vacunados acumulado, para de esta manera llegar a obtener el tiempo de inmunidad de rebaño de 5'483.917 millones de bogotanos vacunados, se deben aplicar 10'967.834 vacunas, aplicando dos dosis.

\section{RESULTADOS}

En la tabla 1 se observa la descripción del modelo ARIMA (p,d,q), para los datos acumulados de vacunación en Bogotá para COVID-19. En la figura 2 podemos apreciar en la línea de color rojo, el valor observado de la dinámica de vacunación para COVID-19 en Bogotá por días desde el 21 de febrero 2021 hasta el 11 de julio 2021 y su respectiva predicción por días desde el 12 de julio 2021 hasta el 6 de diciembre 2021 con la línea azul, con lo cual podemos realizar un pronóstico del acumulado de las personas vacunadas para COVID-19, obtenido según el tiempo. 
Tabla 1. Descripción del modelo ARIMA

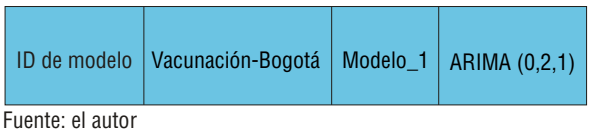

Fuente: el autor

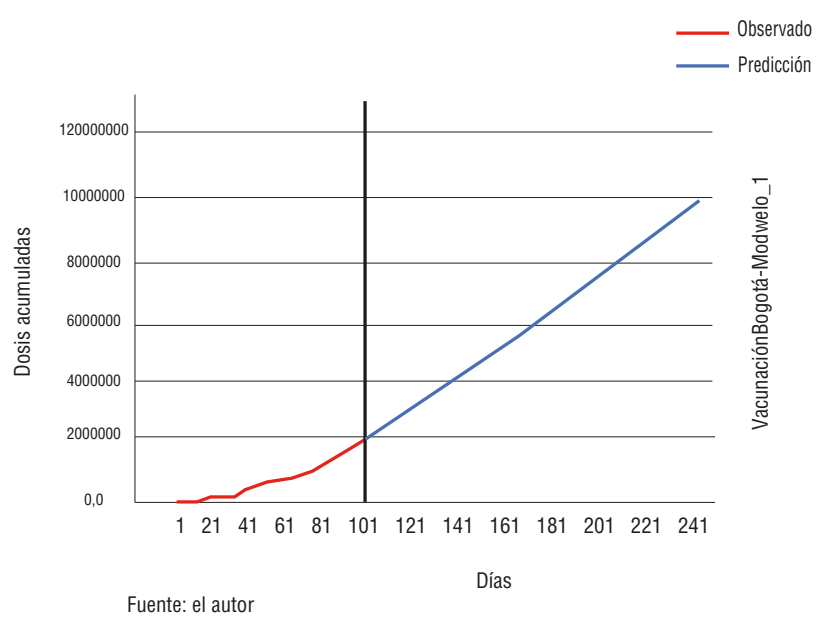

Figura 2. Proyección modelo ARIMA, dosis acumuladas para COVID-19 en Bogotá.

En la figura 3 se describe día a día el acumulado de dosis aplicadas a la población de Bogotá, el día 1 corresponde al 12 de julio 2021 y el día 146 al día 6 de diciembre 2021, con esta información se aprecia que para el 6 de diciembre 2021 se logrará obtener la inmunidad de rebaño, aplicando dos dosis por persona, para esta fecha se habrán aplicado 11'041.306 dosis para inmunizar a la población.

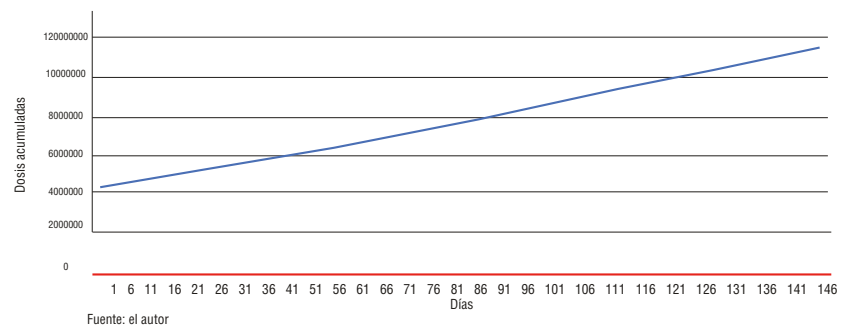

Figura 3. Predicción de dosis acumuladas para COVID-19 en Bogotá

\section{CONCLUSIONES}

Se determinó el pronóstico del estimativo del tiempo para alcanzar la inmunidad de rebaño para COVID-19 en Bogotá. Según el modelo predictivo ARIMA utilizado en esta investigación, arrojó que para el 6 de diciembre 2021 se logrará obtener la inmunidad de rebaño, para esta fecha se habrán aplicado 11'041.306 dosis para inmunizar a la población. Se espera que la efectividad después de dos dosis será algo mayor que con una dosis. Un mayor número de personas se favorecerán de la inmunización al ampliar el intervalo entre dosis en tiempos de carencia de vacunas, brindando a más personas un provecho directo y también la posibilidad de un beneficio indirecto al acrecentar la inmunidad de la población a la enfermedad COVID-19. ${ }^{17}$

De esta investigación se deduce el significativo papel que alcanzan los modelos matemáticos a la hora de simular los procesos de vacunación y de esta manera, se trazan a futuro, vías de investigación en la modelización matemática para lograr la inmunidad de rebaño de cualquier proceso infeccioso.

\section{REFERENCIAS}

1. Organización Mundial de la Salud. Cronología de la respuesta de la OMS a la COVID-19 [Internet]. 2020 [citado 2021 abril 10]; Disponible en: https://www.who.int/es/news-room/detail/2906-2020-covidtimeline

2. Organización Mundial de la Salud. Coronavirus disease (COVID-19) [Internet]. 2021 [citado 2021 abril 10]. Disponible en: https://covid19.who.int/ Salasituacion PBA

3. Silva A, Aguirre M, Ballejo C, Marro M, Gamarnik A, Vargas G, et al. Seroprevalencia de Infección por SARS-COV-2 en PS de la Región Sanitaria VIII, Provincia de Buenos Aires, Argentina. Rev Argent Salud Pública. 2020;12(Suplemento Covid-19):1-9.

4. Creech CB, Walker SC, Samuels RJ. SARS-CoV-2 vaccines. JAMA. 2021;325(13):1318-1320. doi: 10.1001/jama.2021.3199

5. Boland B. ¿Qué es la inmunidad de rebaño? [Internet]. Banner Health; 2020 [citado abril 10 de 2021]. Disponible en: https://www.bannerhealth. com/es/healthcareblog/teach-me/what-is-herd-immunity

6. Cochrane Iberoamérica. Inmunidad de grupo (inmunidad de rebaño) y COVID-19 [Internet]. Colombia: DANE; 2020 [citado 2021 abril 10]; Disponible en: https://es.cochrane.org/es/inmunidad-degrupo-inmunidad-de-reba\%C3\%Blo-y-covid-19

7. Gómez-Lucía E, Ruiz-Santa-Quiteria J. ¿Qué es la inmunidad de rebaño y por qué Reino Unido creía que podía funcionar? [Internet]. Unidad de Cultura Científica y de Divulgación - Universidad Complutense de Madrid; 2020 [citado abril 10 de 2021]. Disponibleen:https://www.ucm.es/otri/noticias-quees-la-inmunidad-de-rebano-y-por-que-reino-unido-cree-que-puede-funcionar

8. Ministerio de Salud y Protección Social. Vacunación contra el COVID-19 [Internet]. Ministerio de Salud y Protección Social; 2021 [citado abril 19 de 2021]; Disponible en: https://www.minsalud.gov. co/salud/publica/Vacunacion/Paginas/Vacunacion-covid-19.aspx

9. Secretaría Distrital de Salud. Vacunación contra el COVID-19 [Internet]. 2021 [citado abril 19 de 2021]; Disponible en: http:// www.saludcapital.gov.co/Paginas2/Inicio.aspx

10. De la fuente S. Modelo ARIMA (p, d, q). Madrid: Universidad Autónoma de Madrid; 2020. p. 53.

11. Calvo D. Análisis de series temporales en R. ARIMA [Internet]. 2018 [citado 2020 noviembre 7]; Disponible en: https://www. diegocalvo.es/analisis-de-series-temporales-en-r-arima/ 
12. Maté C. Modelos ARIMA [Internet]. 2012 [citado 2020 noviembre 7]; Disponible en: https://es2.slideshare.net/juan_churqui/ modelo-arima-14236175?from_action=save

13. Villareal, F. Introducción a los Modelos de Pronósticos [Internet]. 2016 [citado abril 19 de 2021]; Disponible en: https://www. matematica.uns.edu.ar/uma2016/material/Introduccion_a_los_ Modelos_de_Pronosticos.pdf

14. Díaz Pinzón JE. Medidas de frecuencia por COVID-19 en Bogotá DC. Repert Med Cir. 2020;29(Núm. Supl.1):94-98. https://doi. org/10.31260/RepertMedCir.01217372.1110

15. Díaz Pinzón JE. Estimación de las tasas de mortalidad y letalidad por COVID-19 en Colombia. Repert Med Cir. 2020;29(Núm. Supl.1):8993. https://doi.org/10.31260/RepertMedCir.01217372.1103
16. Díaz Pinzón JE. Análisis de los resultados del contagio del COVID-19 respecto a su distribución geográfica en Colombia. Repert Med Cir. 2020;29(Núm. Supl.1):60-64. https://doi.org/10.31260/ RepertMedCir.01217372.1082

17. Luzuriaga J, Mársico F, García E, González V, Kreplak N, Pífano M, González S. Impacto de la aplicación de vacunas contra COVID-19 sobre la incidencia de nuevas infecciones por SARSCOV-2 en PS de la Provincia de Buenos Aires. [Internet]. Scielo; 2021 [citado 18 abr 2021]. Disponible en https://doi.org/10.1590/ SciELOPreprints. 2068 